\title{
Abstracts" \\ 11th International Conference on Developmental Coordination Disorder (DCD11)
}

\section{Developmental coordination disorder and other neurodevelopmental disorders: a focus on comorbidity}

Toulouse, France, July 2-4, 2015

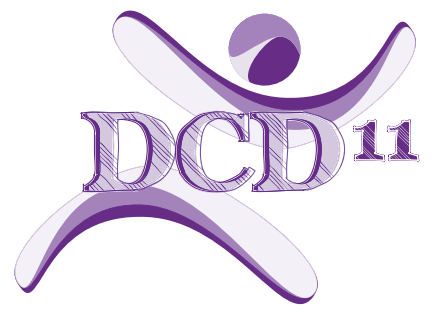

DCD11 Organizing Committee

Jean-Michel Albaret, University of Toulouse; Christophe Barré, University of Toulouse; Maëlle Biotteau, University of Toulouse; Mélody Blais, University of Toulouse; Yves Chaix, Neuropediatric Unit, University Medical Centre of Toulouse; Marianne Jover, Aix-Marseille University; Caroline Karsenty, University Medical Centre of Toulouse; Jennifer LarengArmitage, University of Toulouse; Agnès Laurent, University of Toulouse; Anaïs Mazella, University of Toulouse; Régis Soppelsa, University of Toulouse; Jessica Tallet, University of Toulouse

\section{DCD11 Scientific Committee}

Jean-Michel Albaret, University of Toulouse, France; Christine Assaiante, Aix-Marseille University, France; Anna L. Barnett, Oxford Brookes University, UK; Patrick Berquin, University of Amiens, France; Rainer Blank, University of Heidelberg, Germany; John Cairney, McMaster University, Hamilton, Canada; Chantal Camden, University of Sherbrooke, Canada; Yves Chaix, University of Toulouse, France; Jane E. Clark, University of Maryland, USA; Reint H. Geuze, University of Groningen, The Netherlands; Dido Green, Oxford Brookes University, UK; Sheila E. Henderson, Institute of Education University of London, UK; Marianne Jover, Aix-Marseille University, France; Marie-Laure Kaiser, University Hospital of Lausanne, Switzerland; Mitsuru Kashiwagi, Hirakata City Hospital, Japan; Amanda Kirby, University of Wales, UK; Livia C Magalhães, Federal University of Minas Gerais, Brazil; Anita E. Pienaar, North-West University, South Africa; Helen Polatajko, University of Toronto, Canada; Bouwien Smits-Engelsman, KU Leuven, Belgium; David Sugden, University of Leeds, UK; Jessica Tallet, University of Toulouse, France; Jean-Luc Velay, Aix-Marseille University, France; Hilde van Waelvelde, Artevelde University College \& Ghent University, Belgium; Naomi Weintraub, Hebrew University of Jerusalem, Israel; Peter Wilson, Australian Catholic University, Australia; Jill G. Zwicker, University of British Columbia, Canada

Comorbidity refers to the presence of two or more disorders in the same person (especially DCD, dyslexia and attention deficit hyperactivity disorder in terms of developmental disorders). There has been growing interest in the presence of comorbidity in persons with neurodevelopmental disorders. Many recent studies suggest that up to half of all individuals diagnosed with a psychiatric or neurodevelopmental disorder have more than one condition. Comorbidity not only impacts patient outcomes but can also create a significant strain on both family and school life. It can also complicate diagnosis and healthcare organization. The $11^{\text {th }}$ congress on DCD aimed to address some of the important issues surrounding comorbidity in neurodevelopmental disorders. Three main topics were covered during oral and poster presentations: (1) assessment and diagnostic criteria, (2) underlying processes, causal factors, and prognostic markers, and (3) intervention and management of DCD and associated disorders.

\footnotetext{
"These abstracts have been reproduced as supplied by the authors, without editorial input by the Journal of Comorbidity. Inadequacies in preparation, spelling, grammar, syntax and style are the authors' responsibility. The content reflects the authors' work/opinions and is not necessarily the position of the journal or publisher.
}

Published: June 23, 2015

(c) 2015 The Authors. This is an open-access article distributed under the Creative Commons Attribution-NonCommercial 4.0 License, which permits all noncommercial use, distribution, and reproduction in any medium, provided the original author and source are credited. 
motor control, and Factor III Ball skills. The Little DCDQ-NL had a moderate correlation with the M-ABC2-NL $(r=.551)$ and the EYMSC $(r=-.626)$. The Little DCDQ-NL showed a significantly lower score in the at-risk group $(<16$ th percentile in the $\mathrm{M}-\mathrm{ABC} 2)$ in comparison to the group with age typical development $(\mathrm{p}<.001)$. Most of the parents $(62 \%)$ found the Little DCDQ-NL easy or really easy to fill out, a third found it just right, and $6 \%$ evaluated it as difficult to fill out.

Discussion: In sum, the current Dutch version of the Little DCDQ meets most of the necessary psychometric criteria of an early movement skills screening instrument. Based on the findings, this tool is able to discriminate between young typically developing children and children at risk of DCD References:

Rihtman, T., Wilson, B. N., \& Parush, S. (2011). Development of the Little Developmental Coordination Disorder Questionnaire for preschoolers and preliminary evidence of its psychometric properties in Israel. Research in Developmental Disabilities, 32, 1378-1387.

Keywords: Developmental Coordination Disorder; Little DCDQ; Psychometrics.

\section{Daily motor characteristics in children with Developmental Coordination Disorder and in children with Specific Learning Disorder}

B. Caravale ${ }^{1}$, S. Baldi ${ }^{2}$, F. Presaghi ${ }^{1}$, R. Penge $^{3}$, I. Salvadore ${ }^{3} \&$ M. Nunzi ${ }^{2}$

${ }^{1}$ Department of Developmental and Social Psychology, Sapienza University of Rome, Italy. barbara.caravale@uniroma1.it; ${ }^{2}$ Associazione Melograno Psicologia Cinica e Riabilitazione, Rome, Italy; ${ }^{3}$ Department of Paediatrics and Child Neuropsychiatry, Sapienza University of Rome, Italy

Aim: An association between reading difficulties and coordination problems has been reported in several studies over the last decade. The aim of the present study was to investigate daily motor characteristics in two groups of children with developmental disorders, developmental coordination disorder (DCD) and specific learning disorder (SLD), compared to typically developing controls.

Method: 96 children participated in the study, 33 with diagnosis of DCD, 29 with diagnosis of SLD (excluding children with dysgraphia) and 34 typically developing children. The Italian version of 'Developmental Coordination Disorder Questionnaire' (Wilson et al., 2009 and Caravale et al., 2014) was used for the evaluation of the daily motor characteristics. DCDQ is a 15 -item questionnaire designed to be self-administered by parents of children aged 5-15 years. Parents are asked to answer on a five-point Likert scale when comparing motor performance between their child and peers. The Total DCDQ scores and the three DCDQ subscores of the three groups of children were systematically compared in four distinct ANOVAs. When a significant main effect was found, the Tuckey Post-hoc tests were performed.

Results: The three groups compared with regard to DCDQ total showed significant differences $(F(2,93)=42.3 ; \mathrm{p}<0.01)$ with the DCD group scoring significantly lower than both SLD and controls (both $\mathrm{p}<0.01$ ). With regard to sub-scores, SLD reached significantly lower mean scores than controls in 'Fine motor/hand writing' skills and in the 'General coordination' area (both $\mathrm{p}<0.01$ ) while they obtained significantly higher scores than DCD in 'Control during movement' and 'General coordination' areas (both $\mathrm{p}<0.01$ )

Discussion: Children with DCD showed more difficulties in daily motor performance compared to the other groups. Children with diagnosis of SLD diverged from typically developing children in general coordination skills and fine motor abilities. Our data suggest that although children with DCD present a more impaired global motor development, SLD may highlight difficulties in certain motor areas. These results are consisted with previous studies on motor difficulties in poor readers (Iversen et al., 2005) and encourage the routinely evaluation of motor skills in children with learning problems.

\section{References:}

Caravale, B., Baldi, S., Gasparini, C., \& Wilson, B. N. (2014). Cross-cultural adaptation, reliability and predictive validity of the Italian version of Developmental Coordination Disorder Questionnaire (DCDQ). European Journal of Paediatric Neurology, 18(3), 267-272.

Iversen, S., Berg, K., Ellertsen, B., \& Tønnessen, F. E. (2005). Motor coordination difficulties in a municipality group and in a clinical sample of poor readers. Dyslexia, 11(3), 217-231.

Wilson, B. N., Crawford, S. G., Green, D., Roberts, G., Aylott, A., \& Kaplan, B. J. (2009). Psychometric properties of the revised developmental coordination disorder questionnaire. Physical \& Occupational Therapy in Pediatrics, 29(2), 182-202.

Keywords: Parent questionnaire; Motor characteristics; DCD; Specific learning disorder.

Little DCDQ-US in typically developing children and children with motor concerns with and without ASD

\section{S.A. Cermak ${ }^{1} \&$ A. Foran Jozjkowski ${ }^{2}$}

${ }^{1}$ Department of Occupational Science and Occupational Therapy, University of Southern California, Los Angeles, California, USA. sharon.cermak@gmail.com; 2Department of Occupational Therapy, Towson University, Towson, MD, USA.

Aim: To examine whether there are differences between children with motor concerns with and without a diagnosis of ASD on the Little Developmental Coordination Disorder Questionnaire- US.

Method: Participants included children ages three and four years old, 85 children who were typically developing: 75 children who were receiving services that included motor concerns. The inclusion criteria for children with motor concerns included having gross motor or/and fine motor skills below average based on parent or therapist report and receiving one or more services of the following services related to motor needs: speech therapy, occupational therapy or physical therapy, psychological services. These children were not required to have a diagnosis of DCD; it was sufficient for children to have a general complaint of motor developmental problems. Children diagnosed with neurological or physical impairment such as cerebral palsy or muscular dystrophy, were excluded. Children with suspected or diagnosed ASD were not excluded. Of the 75 children with motor concerns, 27 parent reported that their child had a confirmed or suspected diagnosis of ASD Parents completed the Little Developmental Developmental Coordination Disorder Questionnaire-US (LDCDQ-US), a 15 item questionnaire designed to screen young children at risk for DCD.

Results: There were significant differences between the group receiving services for motor concerns $(n=75)$ and the typically developing group $(n=85)$. For the total score, the three component scores (Control during movement; Fine motor: General coordination), and the individual items (all $\mathrm{p}<.001)$. Of the group with motor concerns, there were no significant differences between children with and without ASD.

Discussion: The Little DCDQ-US distinguishes between children with and without motor concerns but does not distinguish between children with motor concerns with and without ASD; both groups show impairments in motor performance based on parent-report. This finding is consistent with other investigators who have reported that children with ASD have motor concerns similar to children with DCD.

Keywords: Developmental Coordination Disorder; Autism Spectrum Disorder; Questionnaire.

Motor impairment in children with Language Disorders: Influence of the language profile

Y. Chaix ${ }^{1,2}$, L. Beringer ${ }^{2}$, N. Faure Marie $^{2}$, C. Chignac ${ }^{2}$, I. Barry ${ }^{2}$, C. Karsenty $^{2} \&$ J.-M. Albaret ${ }^{3}$

${ }^{1}$ Inserm; Imagerie Cérébrale et Handicaps Neurologiques UMR 825; CHU Purpan, Place du Dr Baylac, F-31059 Toulouse Cedex 9, France. 